\title{
The NO $\left(X^{2} \Pi\right)-$ Ne complex. II. Investigation of the lower bound states based on new potential energy surfaces
}

\author{
Millard H. Alexander ${ }^{\mathrm{a})}$ \\ Department of Chemistry and Biochemistry, University of Maryland, College Park, Maryland 20742-2021 \\ Pavel Soldán \\ Department of Chemistry, University of Durham, South Road, Durham DH1 3LE, United Kingdom \\ Timothy G. Wright \\ School of Chemistry, Physics and Environmental Science, University of Sussex, Falmer, Brighton, \\ BN1 9QJ, United Kingdom \\ Yangsoo Kim and Henning Meyer \\ Department of Physics and Astronomy, University of Georgia, Athens, Georgia 30602-2451
}

Paul J. Dagdigian

Department of Chemistry, The Johns Hopkins University, Baltimore, Maryland 21218-2685

Edmond P. F. Lee

Department of Chemistry, University of Southampton, Highfield, Southampton, SO17 1BJ,

United Kingdom and Department of Applied Biology and Chemical Technology,

Hong Kong Polytechnic University, Hung Hom, Hong Kong

(Received 25 September 2000; accepted 19 December 2000)

\begin{abstract}
High-quality $a b$ initio potential energy surfaces were calculated and subsequently used to predict the positions of the lowest bend-stretch vibrational states of the $\mathrm{NO}\left(X^{2} \Pi, v=0\right)-\mathrm{Ne}$ complex. The vibrational wavefunctions and basis set expansion coefficients, determined within the adiabatic bender model, were then used to simulate the observed spectrum for excitation of the $\mathrm{NO}\left(X^{2} \Pi, v=2\right)-\mathrm{Ne}$ complex. The overall position and rotational substructure matches well the experimental results for this system, which are presented in the preceding article [Y. Kim, J. Fleniken and H. Meyer, J. Chem. Phys. 114, 5577 (2001)]. A heuristic Hamiltonian, which includes the most important couplings and splittings, is used to improve the fit to experiment. (C) 2001
\end{abstract} American Institute of Physics. [DOI: 10.1063/1.1349086]

\section{INTRODUCTION}

Collisions of diatomic free radicals with noble gases provide exemplary systems for the investigation of interactions of molecular free radicals, which are of great importance in all of chemical kinetics. These interactions are complicated by the presence of electronic spin and/or orbital angular momentum, which can couple with the orbital angular momentum of the collision partners. ${ }^{1}$ Collisions of the NO molecule in its $X^{2} \Pi$ electronic ground state with noble gases have been the paradigm. Although the $\mathrm{NO}-\mathrm{Ar}$ and $\mathrm{NO}-\mathrm{He}$ systems have been extensively studied, there has been relatively little attention given to the $\mathrm{NO}-\mathrm{Ne}$ system. In early work Thuis et al. used total scattering experiments to investigate the isotropic portion of the NeNO potential. ${ }^{2}$

Spectroscopic investigation of the bound states of the weakly bound complex between a molecular radical and a noble gas atom provides information on the shape of the potential energy surfaces (PESs) in the region of the van der Waals minimum, information which complements that available from inelastic scattering studies. ${ }^{3}$ This has been exemplified by work over the past decade on the complexes of $\mathrm{OH}\left(X^{2} \Pi\right)$ with $\mathrm{Ar}$ and $\mathrm{Ne}^{4-11}$ For NO-Ar and NO-Ne

a)Electronic mail: mha@mha-ibm4.umd.edu
Mills, Western, and Howard ${ }^{12,13}$ used microwave and radiofrequency spectroscopy to investigate transitions between a number of multiplet levels associated with the lowest rotational levels of the complex. This past year, Meyer and co-workers ${ }^{14}$ have used overtone pumping in the region of the NO $v=2 \leftarrow 0$ stretch, followed by resonance enhanced multiphoton ionization (REMPI) detection, to observe rotationally resolved spectra corresponding to the ground and first excited ro-vibronic states of the $\mathrm{NO}(X)-\mathrm{Ar}$ complex.

In earlier work, Miller and Cheng ${ }^{15}$ and Meyer and co-workers ${ }^{16}$ used $(2+1)$ REMPI to investigation the complex of NO with Ne by making use of several different NO Rydberg states. In the preceding article ${ }^{17}$ Kim, Meyer, and Fleniken (KMF) have used this technique to carry out the IR (infrared) REMPI double resonance spectroscopy of the NO-Ne complex and hence extract information on the lowest bend-stretch levels of this complex, in a manner similar to that described earlier for $\mathrm{NO}-\mathrm{Ar} .{ }^{18}$

Because of the simplicity of the NO-Ne system, and encouraged by the success of our calculations ${ }^{18}$ of the bound states of the NO-Ar complex, we undertook a theoretical investigation of the lower bound states of the NO-Ne complex. We report in the next section the calculation of highquality $a b$ initio potential energy surfaces, which are subse- 
quently used to calculate the bound states of the complex based on a full close-coupled expansion of the bound-state wavefunction.

The organization of this article is as follows: In the next section we review briefly the NeNO PESs used. The method and computational details for the determination of the bound states of the $\mathrm{NO}(X)-\mathrm{Ne}$ complex are described in Secs. III and IV. This is followed by introduction of the heuristic Hamiltonian invoked in the preceding article. ${ }^{17}$ The formalism used to simulate the excitation spectra is presented in Sec. VI. Section VII contains a comparison of the predicted and experimental spectra. We end with a brief conclusion.

\section{POTENTIAL ENERGY SURFACES}

The approach of a structureless atom to a molecule in a ${ }^{2} \Pi$ electronic state gives rise to two PESs, of $A^{\prime \prime}$ and $A^{\prime}$ reflection symmetry. ${ }^{19}$ The PESs are a function of the three Jacobi coordinates $r$ (the NO bond distance), $R$ (the distance between the $\mathrm{Ne}$ atom and the center of mass of the NO molecule), and $\theta$ (the angle between $\mathbf{r}$ and $\mathbf{R}$ ) with $\theta=0$ corresponding to colinear Ne...NO. Our first task was the $a b$ initio determination of these two PESs.

A grid of potential energy points was calculated by restricted coupled-cluster RCCSD $(\mathrm{T})$ calculations ${ }^{20}$ using an aug-cc-pVQZ basis with the MOLPRO 98.1 ab initio program suite. $^{21}$ [It is acknowledged that there is a small error in the calculation of $\operatorname{RCCSD}(\mathrm{T})$ energies in this version of the program; however, subsequent testing of the size of the error using MOLPRO $2000^{21}$ showed that the error would leave the calculated quantities in the present article essentially unchanged.] The grid of geometries used was selected on the results of geometries optimized at the UQCISD level using GAUSSIAN $98,{ }^{22}$ with a higher concentration of points around the minimum. All points were corrected for basis set superposition error using the full counterpoise method of Boys and Bernardi. ${ }^{23}$ To reduce the total computer time required, the NO bond distance was held fixed at $1.1526 \AA$ [the equilibrium internuclear separation predicted by a comparable RCCSD(T) calculation on the isolated NO molecule].

The center-of-mass distances, $R$, selected were $2.3,2.8$, 3.1, 3.3, 3.5, 3.6, 3.7, 3.8, 4.0, 4.5, 5.0, and 7.0 $\AA$, while the Jacobi angles, $\theta$, chosen were 180.0, 154.126, 132.624, 111.292, 90.0, 68.708, 47.376, 25.874, and 0.0 degrees. These values are the nodes for the Gauss-Lobatto quadrature based on the spectral representation of the Legendre recursive system. ${ }^{24}$ The ORTHOPOL program suite ${ }^{24}$ was used to determine the nodes and corresponding weights.

For both the $A^{\prime}$ and $A^{\prime \prime}$ PESs a function reciprocalpower reproducing kernel Hilbert space (RP-RKHS) interpolation procedure ${ }^{25,26}$ was used to construct an analytic approximation to the dependence on $R, V\left(R, \theta_{i}\right)$, for each separate value of $\theta_{i}$. To obtain the correct long-range behavior of the interaction potential, ${ }^{27}$ interpolation with respect to $R^{2}$ was performed using the RP-RKHS parameters $m=2$ and $n=2$. In the treatment of the nuclear motion of the NeNO complex, it is convenient to work with the average and halfdifference of the $A^{\prime \prime}$ and $A^{\prime}$ PESs, defined as ${ }^{19}$

$$
V_{\text {sum }}(R, \theta)=0.5\left[V_{A^{\prime \prime}}(R, \theta)+V_{A^{\prime}}(R, \theta)\right]
$$
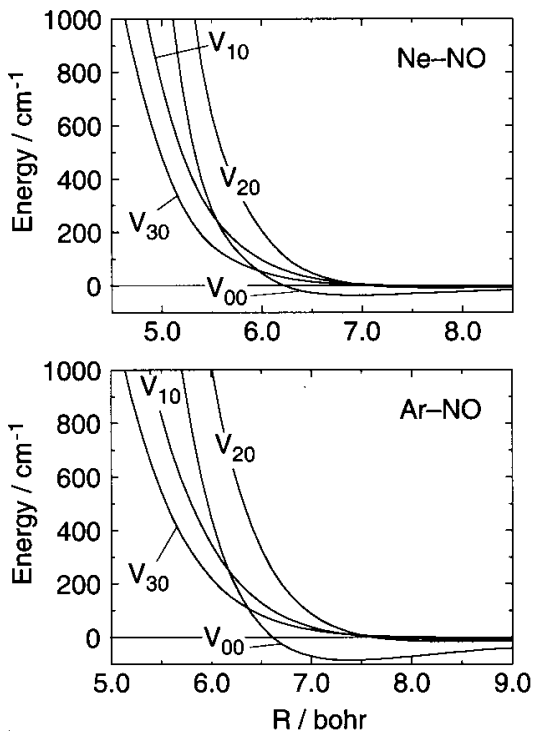

FIG. 1. Comparison of the dependence on the Ne/Ar-NO center-of-mass separation of the largest of the $V_{l 0}(R)$ terms in the Legendre expansion of the sum PES [Eq. (2a)] as predicted by our $\operatorname{CCSD}(\mathrm{T})$ calculations. The ArNO results are taken from Ref. 29.

and

$$
V_{\text {dif }}(R, \theta)=0.5\left[V_{A^{\prime \prime}}(R, \theta)-V_{A^{\prime}}(R, \theta)\right] .
$$

Finally, in order to obtain the $\theta$ dependence for a given value of $R$, the sum and difference potentials were projected onto reduced rotation matrix elements, ${ }^{28}$ making use of the Gauss-Lobatto quadrature: ${ }^{24}$

$$
V_{\text {sum }}(R, \theta)=\sum_{l=0}^{l_{\max }} V_{l 0}(R) d_{00}^{l}(\theta)
$$

and

$$
V_{\text {dif }}(R, \theta)=\sum_{l=2}^{l_{\max }} V_{l 2}(R) d_{20}^{l}(\theta),
$$

where $l_{\max }=8$, corresponding to the number of angles in the ab initio grid.

The dependence on $R$ of the largest of the $V_{l 0}(R)$ and $V_{l 2}(R)$ expansion terms is illustrated in Figs. 1 and 2, for the RCCSD(T) NeNO calculations of the present article. These are compared with similar plots for the UCCSD(T) ArNO PESs. ${ }^{29}$ The overall behavior is very similar, except for a reduction of $\sim 0.5 \mathrm{bohr}$ in the size of the potential, which reflects the smaller size of the $\mathrm{Ne}$ atom, and a smaller well. The well depth $\left(D_{e}\right)$ in the isotropic term $\left[V_{00}(R)\right]$ in the expansion is $35.3 \mathrm{~cm}^{-1}$ and the minimum occurs at 6.93 bohr. These values agree reasonably well with the empirical estimates of Thuis et al. from molecular beam scattering experiments $\left(\varepsilon \cong 50 \mathrm{~cm}^{-1}, r_{e} \cong 5.9 \mathrm{bohr}\right){ }^{2}$

For both ArNO and NeNO the difference potential is negative. This indicates that the $A^{\prime \prime}$ PES is less repulsive than the $A^{\prime}$ PES. As might be expected, the magnitude of the difference potential is roughly a factor of 2 smaller for the $\mathrm{NeNO}$ system. In contrast, for the HeNO system at some geometries the $A^{\prime \prime}$ PES lies above the $A^{\prime}$ PES. ${ }^{30,31}$ We refer 

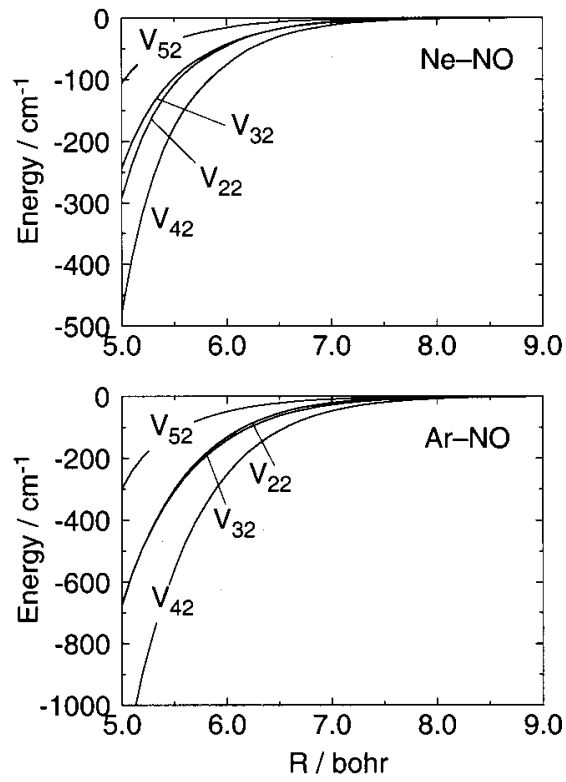

FIG. 2. Comparison of the dependence on the Ne/Ar-NO center-of-mass separation of the largest of the $V_{l 2}(R)$ terms in the Legendre expansion of the difference PES [Eq. (2b)] as predicted by our $\operatorname{CCSD}(\mathrm{T})$ calculations. The ArNO results are taken from Ref. 29.

the reader to the earlier work of Wright, Chalasinski, and co-workers for a more complete discussion of the different potentials for HeNO. ${ }^{30,31}$

\section{BOUND STATES OF THE NO-Ne COMPLEX: FORMALISM}

To describe the bound levels of the NO-Ne complex, we expand the complete wavefunction in a basis formed by products of the wavefunctions which describe the electronicrotational states of the NO molecule and angular momentum functions which describe the orbital (end-over-end) rotation of the complex. One can use a space- or body-frame expansion. ${ }^{18}$

In a body-frame $(\mathrm{BF})$ expansion we construct the complete wavefunction by first defining primitive- $P \omega$ functions, which are, in the coordinate representation,

$|j P \omega J M\rangle=\frac{[(2 j+1)(2 J+1)]^{1 / 2}}{4 \pi} D_{M P}^{J *}(\hat{R}) D_{P \omega}^{j *}\left(\hat{r}_{b}\right)|\lambda \sigma\rangle$.

Here $j$ denotes the total angular momentum of the diatomic molecule, with projections $P$ and $\omega$ along, respectively, $\mathbf{R}$ and $\mathbf{r}$, the body- and molecule-fixed $z$-axes. The subscript $b$ indicates that the angles which define the orientation of $\mathbf{r}$ are taken with respect to the body frame. Also, $|\lambda \sigma\rangle$ designates the electronic component of the wavefunction, in which $\lambda$ and $\sigma$ denote, respectively, the molecule-frame projections of the electronic orbital and spin angular momenta. Note also that $\omega=\lambda+\sigma$, and $P$ is the projection of both $j$ and $J$ along R. Definite-parity combinations of these primitive functions can be defined

$$
|j P \omega \xi J M\rangle=2^{-1 / 2}[|j P \omega J M\rangle+\xi|j,-P,-\omega J M\rangle],
$$

where the symmetry index $\xi$ can take on the values \pm 1 . The total parity of the wave functions defined in Eq. (4) is $\xi$ $(-1)^{J-1 / 2}$. We designate these functions signed-P $\omega$ basis functions, because the two terms correspond to a definite sign of the product $P \times \omega$. Alternative choices for the BF basis in which to expand the total wavefunction have been discussed previously. ${ }^{18}$

We expand the full wavefunction in this signed- $P \omega \mathrm{BF}$ basis [Eq. (4)]. This gives rise to the standard set of closecoupled (CC) equations for the expansion coefficients. In matrix notation these are

$$
\left[\frac{d^{2}}{d R^{2}}+W(R)\right] C^{J}(R)=0
$$

where $W(R)$ is the sum of three matrices: the diagonal wavevector matrix, the matrix of the square of the orbital angular momentum $(\mathbf{L}=\mathbf{J}-\mathbf{j})$, and the matrix of the interaction potential(s). ${ }^{29,32}$ Expressions for the latter have been given previously. ${ }^{19,33}$

In the determination of the matrix elements of $\mathbf{L}^{2}$ we assume a pure-precession limit, in which the electronic orbital angular momentum is unchanged from its asymptotic value. In addition, in the determination of the internal energy levels of each of the basis states (in either the body- or spaceframe) we assume that the spin-orbit splitting in the NO molecule does not change as the $\mathrm{Ne}$ atom approaches. Both of these approximations are likely well justified for the weakly bound NO-Ne complex, where the electronic structure of the NO moiety remains essentially unperturbed.

In the BF expansion the potential matrix is block diagonal in $P$ and independent of $J$ and the sign of $P$. The average $\left(V_{\text {sum }}\right)$ term in the expansion of the potential [Eq. (2a)] couples states with the same value of the projection $\omega$, while the difference $\left(V_{\mathrm{dif}}\right)$ term mixes states with $\omega^{\prime}=\omega \pm 2$. The matrix of the orbital (end-over-end) rotation of the complex has both diagonal and off-diagonal terms. In the centrifugaldecoupling (CD) approximation, ${ }^{34,35}$ the off-diagonal terms, which reflect Coriolis coupling between states which differ in either the magnitude or sign of $P$, are neglected.

Additional physical insight can be gained by an examination of the definite- $P$ adiabatic energies, obtained by diagonalization of the unique block of the $\mathrm{BF} W(R)$ matrix corresponding to the chosen value of $P$. The definite- $P$ adiabatic energies are defined by

$$
w_{P}(R)=T(R) W_{P}(R) T(R)^{T},
$$

where $T(R)$ is the matrix which diagonalizes the $P$ th block of the $\mathrm{BF} W(R)$ matrix. The $R$ dependence of the $w_{P}(R)$ adiabatic energies define "adiabatic bender" (AB) potentials. These can then be used to investigate the vibrational motion along the van der Waals stretching coordinate $R .{ }^{32-34}$ To the extent that off-diagonal matrix elements of the radial kinetic energy operator can be ignored, and the Coriolis couplings are small, the energies of these vibrational levels will provide a good first approximation to the bend-stretch states of the NeNO complex. ${ }^{36-38}$ 


\section{BOUND STATES OF THE NO-Ne COMPLEX: PERTURBATION MODEL}

Alternatively, one can use simple arguments based on perturbation theory to obtain an understanding of the energy levels of the complex. ${ }^{34,35,39}$ The coupling between individual $|j P \omega \xi J M\rangle$ states due to the various terms in the expansion of both $V_{\text {sum }}$ and $V_{\text {dif }}$ is given by ${ }^{35}$

$$
\begin{aligned}
\left\langle j P \omega \xi J M|V| j^{\prime} P^{\prime} \omega^{\prime} \xi^{\prime} J M\right\rangle \\
=\delta_{P P^{\prime}} \delta_{\xi \xi^{\prime}}\left[(2 j+1)\left(2 j^{\prime}+1\right)\right]^{1 / 2} \\
\quad \times(-1)^{P-\omega} \sum_{l}\left(\begin{array}{ccc}
j & l & j^{\prime} \\
-P & 0 & P
\end{array}\right) \\
\quad \times\left(\begin{array}{ccc}
j & l & j^{\prime} \\
-\omega & \lambda-\lambda^{\prime} & \omega^{\prime}
\end{array}\right) V_{l, \lambda-\lambda^{\prime}}(R),
\end{aligned}
$$

where (:::) is a Wigner $3 j$ symbol. $^{28,40}$ The electrostatic interaction splits the free rotor levels (for a given $j$ ) into sublevels characterized by the product $P \omega$. Since $P$ is restricted to positive values, we expect $j+\frac{1}{2}$ different $P$ levels. These levels will be split additionally into pairs corresponding to the sign of $\omega$. This results in a total of $2 j+1$ levels. These are further degenerate in the symmetry index $\xi$.

To first order the energies of the $|j P \omega \xi J M\rangle$ states will be given by the diagonal elements of Eq. (7). For a given value of $P$, the contribution of the sum potential is represented by the terms with $\lambda=\lambda^{\prime}$. The diagonal matrix elements for $\omega$ positive and for $\omega$ negative will be related by the symmetry of the second $3 j$ symbol in Eq. (7). Consequently, the matrix elements of the potential which are diagonal in $j$ obey the relation

$$
\begin{aligned}
& (-1)^{-\omega}\left(\begin{array}{ccc}
j & l & j \\
-\omega & 0 & \omega
\end{array}\right) V_{l 0}(R) \\
& =(-1)^{2 j+l}(-1)^{\omega}\left(\begin{array}{ccc}
j & l & j \\
\omega & 0 & -\omega
\end{array}\right) V_{l 0}(R) .
\end{aligned}
$$

Since both $\omega$ and $j$ are half integer, it follows that only the odd terms in the sum potential will split the $+\omega$ and $-\omega$ levels, by an equal and opposite amount. Thus, to first order, the rotational levels with $\omega$ positive and $\omega$ negative will be split by the matrix elements which are diagonal in $j$. Again to first order, this splitting will be independent of $J$, since the matrix elements in Eq. (7) are independent of $J$.

As discussed in Sec. III, the matrix of the square of the orbital angular momentum $\mathbf{L}$ is not diagonal in $P$. Evaluating the matrix elements of $\mathbf{L}^{2}$, and making explicit use of the fact that the ladder operators $J_{ \pm}$are defined with respect to the body frame and hence obey anomalous commutation relations,${ }^{41}$ we find

$$
\begin{aligned}
& B\left\langle j P \omega \xi J M\left|L^{2}\right| j^{\prime} P \omega^{\prime} \xi^{\prime} J M\right\rangle \\
& \quad=\delta_{j j^{\prime}} \delta_{\xi \xi^{\prime}} B\left[J(J+1)+j(j+1)-2 P^{2}\right]
\end{aligned}
$$

and

$$
\begin{aligned}
B\left\langle j P \omega \xi J M\left|L^{2}\right| j^{\prime} P^{\prime} \omega^{\prime} \xi^{\prime} J M\right\rangle & \\
= & \delta_{j j^{\prime}} \delta_{\xi \xi^{\prime}} B\left[F\left(P^{\prime}\right) \delta_{P P^{\prime}+1} \delta_{\omega \omega^{\prime}}\right. \\
& +F\left(P^{\prime}-1\right) \delta_{P P^{\prime}-1} \delta_{\omega \omega^{\prime}}+\xi F\left(P^{\prime}-1\right) \\
& \left.\times \delta_{-P, P^{\prime}-1} \delta_{\omega,-\omega^{\prime}}\right]
\end{aligned}
$$

where $B=1 / 2 \mu R^{2}$ and

$$
F(P)=\{[J(J+1)-P(P+1)][j(j+1)-P(P+1)]\}^{1 / 2} .
$$

Since the last term in Eq. (10) is the only term which depends explicitly on the value of $\xi$, it is this term which gives rise to a splitting between the positive and negative parity levels. Because of the Kronecker deltas in the last term, this $P$-type doubling will contribute (at third order in perturbation theory $)^{39}$ only for $P=P^{\prime}=\frac{1}{2}$ and $\omega=-\omega^{\prime}$. Green and Lester $^{39}$ showed that this $P$-type doubling is due to a mixed contribution involving the difference potential and the Coriolis coupling. For levels with $P=\frac{3}{2}$ (or higher), the $P$ doubling will appear in fifth order. ${ }^{34}$ Because of the dependence of $F(P)$ on $J$, the magnitude of the $P$-type doubling can be expanded in powers of $\left(J+\frac{1}{2}\right)$. Further, because of the condition that $\omega=-\omega^{\prime}$, the effective size of the $P$-type doubling will be sensitive to the difference potential. The effect of the $\omega$ - and $P$-type doubling is illustrated schematically in Fig. 11 of the preceding article. ${ }^{17}$

The simple, perturbation-theory arguments from the preceding paragraph suggest that a good approximation to the vibration-rotation levels of the $\mathrm{NO}-\mathrm{Ne}$ and $\mathrm{NO}-\mathrm{Ar}$ complexes is

$$
\begin{aligned}
E_{v_{b} v_{s} P \omega \xi}^{J}= & E_{v_{b} v_{s} P}+B J(J+1)-D[J(J+1)]^{2}+\cdots \\
& -\frac{\omega}{|\omega|}\left\{V_{0}+V_{1}\left(J+\frac{1}{2}\right)+V_{2}\left(J+\frac{1}{2}\right)^{2}+\cdots\right. \\
& \left.+\xi\left[C_{0}+C_{1}\left(J+\frac{1}{2}\right)\right]\right\} .
\end{aligned}
$$

The first term represents the vibrational energy of the complex as well as the rotational energy contribution $A_{\text {eff }} P^{2}$ due to the rotation around the $a$-axis. Therefore, the bound state energies of the complex resemble symmetric top levels split by electrostatic interactions and by electronic Coriolis coupling. In first order, the average potential causes an electrostatic splitting $V_{0}$, which removes the degeneracy in $\omega$. The coefficients $V_{i}(i=1,2,3, \ldots)$ parametrize the $J$ dependence of the $\omega$-splitting which are caused by higher-order interactions involving Coriolis coupling and the difference potential. Consequently, these terms are not present if the bound states are calculated within the $\mathrm{CD}$ approximation. For $\mathrm{NO}-\mathrm{Ar}$, the $J$ dependence is dominated by the linear term $\left(V_{1}\right)$, while, for $\mathrm{NO}-\mathrm{Ne}$, we find that only the terms corresponding to even powers of $\left(J+\frac{1}{2}\right)$ contribute. Finally the $P$-type doubling is described by the constants $C_{0}$ and $C_{1}$. We find different parity splittings for the two $\omega$ levels in the NO-Ne complex, in contrast to the $\mathrm{NO}-\mathrm{Ar}$ complex. 


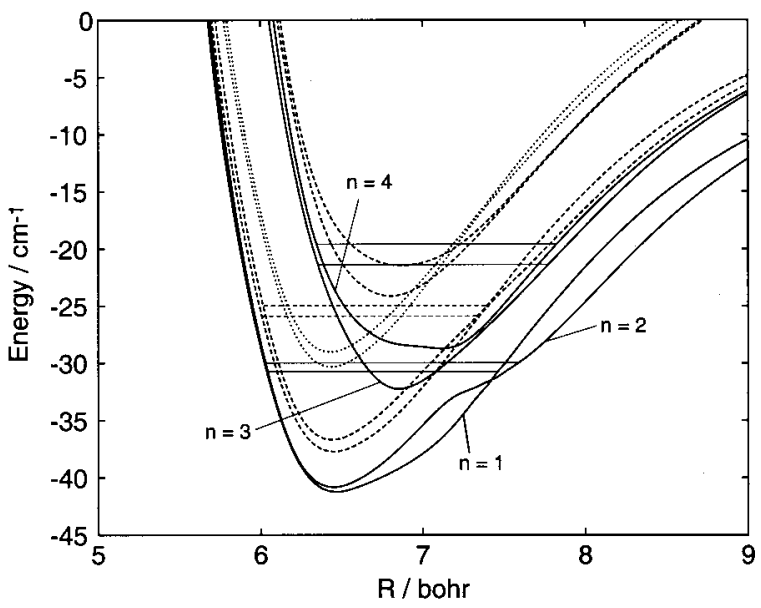

FIG. 3. Adiabatic bender potentials for the NO-Ne complex. The solid and dashed curves correspond, respectively, to $P=\frac{1}{2}$ and $P=\frac{3}{2}$. The states are labeled by $n$, which corresponds to the $n$th eigenvector of the $P$ th block of the BF $W(R)$ matrix [Eq. (6)]. The position of the lowest vibrational level in each $\mathrm{AB}$ potential is shown by the horizontal lines; these indicate the energies of the states listed in Table I.

\section{BOUND STATES OF THE NO-Ne COMPLEX: RESULTS}

Figure 3 shows the $\mathrm{AB}$ potentials for the $\mathrm{NO}-\mathrm{Ne}$ complex as described by the RCCSD(T) PESs. The curves occur in closely spaced pairs, which correspond to the $\omega$-doublets discussed in the preceding section and shown schematically in Fig. 11 of the preceding article. ${ }^{17}$ This assignment is confirmed by examination of the columns of the $T(R)$ matrix in Eq. (6), which are the $A B$ eigenfunctions. In the region of the minima in the curves, the eigenfunctions are dominated by a single signed- $P \omega$ state as defined by Eq. (4). Each of the $\mathrm{AB}$ potentials then corresponds, also, to a single signed$P \omega$ state. Because the minima in both NeNO PESs occur in nearly T-shaped geometry, the states with positive and negative values of $P \times \omega$ lie very close in energy (see Fig. 4). ${ }^{18}$ The $P$-type doubling, also discussed in the preceding section, leads to an additional splitting of each curve (see Fig. 11 of Ref. 17), which is too small to be resolved in Fig. 3.

The energies of the lower bound states of the $\mathrm{NO}-\mathrm{Ne}$ complex were determined variationally. We followed our earlier ${ }^{18,37,42,43}$ implementation of the distributed Gaussian method of Hamilton and Light. ${ }^{44}$ All calculations were carried out with our Hibridon 4 code. $^{45}$

Table I presents the energies of the lower states of the NO-Ne complex for $J=0.5$ and 1.5 , relative to the lowest state, as predicted by the $\mathrm{CC}, \mathrm{CD}$, and $\mathrm{AB}$ calculations with our RCCSD(T) PESs. The absolute energy of the lowest vibrational level defines the zero-point-corrected dissociation energy of the complex, which is $29.35 \mathrm{~cm}^{-1}$. The excellent agreement between the $\mathrm{CD}$ and $\mathrm{CC}$ level energies indicates that $P$ is indeed a good quantum number.

As discussed in the preceding section, in connection with Eq. (10), coupling between levels of different $P$, which is neglected in the $\mathrm{CD}$ approximation, arises from the Coriolis term in the rotational Hamiltonian, which couples directly the states with $P^{\prime}=P \pm 1$. Since this will mix directly states with $P=\frac{1}{2}$ and $P=-\frac{1}{2}$, the $P$-type doubling between the nominally $P=\frac{1}{2}$ states in Table I is much larger than the $P$-doubling between the nominally $P=\frac{3}{2}$ states, which are Coriolis mixed only in fifth order. ${ }^{34}$ The $\mathrm{AB}$ energies also agree very well with the $\mathrm{CC}$ and $\mathrm{CD}$ energies, except in a few cases where residual nonadiabatic coupling occurs between two (or more) AB states.

The details of the generation and spectroscopic investigation of the NO-Ne complexes are described in full detail in the accompanying article. ${ }^{17}$ The rotationally resolved infrared spectrum of the complex associated with the first overtone NO transition is measured in an IR-UV double resonance experiment. Four bands, located at 3724.02, 3727.85, 3732.56 , and $3739.20 \mathrm{~cm}^{-1}$, were detected. Following Kim et al. ${ }^{17}$ we label these bands A, B, C, and D in terms of increasing frequency. For reference, the band origin of the vibrational fundamental in the isolated NO molecule is $3723.85 \mathrm{~cm}^{-1.46}$

Based on the energy level positions listed in Table I, we assign band $\mathrm{A}$ to the lowest vibrational level of the complex $\left(v_{b}, v_{s}\right)=(0,0)$ or, in adiabatic bender notation (Sec. IV), $P=\frac{1}{2}$ and $n=1,2$, while band $\mathrm{B}$ corresponds to excitation of the $(1,0)$ [or $\left.P=\frac{3}{2}, n=1,2\right]$ levels. Band $\mathrm{C}$ corresponds to excitation of the $P=\frac{1}{2}, n=3,4$ levels, and, finally, band $\mathrm{D}$ corresponds to excitation of the $P=\frac{1}{2}, n=1,2$ levels with excitation of one quantum of the van der Waals stretching mode $\left(v_{s}=1\right)$.

With a standard least-squares procedure, the calculated energy levels could be fit extremely well by the heuristic expression derived from perturbation theory arguments, Eq. (12). The residual deviations are $\sim 0.01 \mathrm{~cm}^{-1}$, which is comparable to the precision of the bound-state calculations. Tables II-IV give the values of the constants in Eq. (12) which correspond to the best fit.

\section{INTENSITY FACTORS}

In addition to calculated rovibrational energies, presented in Sec. IV, simulation of the spectrum of the $\mathrm{NO}(X)-\mathrm{Ne}$ complex requires intensity factors, which are described in this section. The calculation of intensity factors for optical transitions in a van der Waals complex of an open-shell diatomic molecule has been discussed previously, ${ }^{35,42}$ and was recently applied to the analogous $\mathrm{NO}(X)-\mathrm{Ar}$ complex. ${ }^{18}$ Consequently, we give only a concise summary here.

Since the AB approximation was shown in the previous section to be reasonably accurate, we express the expansion coefficients in Eq. (5) as

$$
C_{j \omega}^{P N}(R)=\chi_{v}^{P n}(R) w_{j \omega}^{P n}(R),
$$

where $\chi_{v}^{P n}(R)$ is the one-dimensional $v$ th vibrational wave function of the $n$th $\mathrm{AB}$ potential of projection quantum number $P$, and $w_{j \omega}^{P n}(R)$ is the expansion coefficient of state $|j P \omega\rangle$ in the $n$th eigenvector of the $W_{P}(R)$ matrix of Eq. (6).

The chromophore of the transitions reported here is the vibrational transition matrix element within the ground electronic state $\mu_{20} \equiv\langle v=2|\mu| v=0\rangle$, which lies along the NO internuclear axis. The space-fixed components of the electric dipole operator and their matrix elements within the signed- 
TABLE I. Relative energies (in $\mathrm{cm}^{-1}$ ) of lowest bound-states of the NO-Ne complex.

\begin{tabular}{|c|c|c|c|c|c|c|}
\hline \multirow[b]{2}{*}{$P^{\mathrm{d}}$} & \multirow[b]{2}{*}{$n^{\mathrm{e}}$} & \multirow[b]{2}{*}{$v_{s}{ }^{\mathrm{f}}$} & \multicolumn{2}{|c|}{$\mathrm{CC}^{\mathrm{a}}$} & \multirow[b]{2}{*}{$\mathrm{CD}^{\mathrm{b}}$} & \multirow[b]{2}{*}{$\mathrm{AB}^{\mathrm{c}}$} \\
\hline & & & $\pi^{\mathrm{g}}=+1$ & $\pi=-1$ & & \\
\hline \multirow{8}{*}{$\frac{1}{2}$} & & & \multicolumn{4}{|c|}{$J=0.5$} \\
\hline & 1 & 0 & 0 & 0.007 & 0 & 0 \\
\hline & 2 & 0 & 0.86 & 0.84 & 0.76 & 0.76 \\
\hline & 3 & 0 & 7.72 & 7.70 & 7.46 & 9.36 \\
\hline & 4 & 0 & 9.19 & 9.22 & 9.07 & 11.15 \\
\hline & 1 & 1 & 14.66 & 14.41 & 14.18 & 16.84 \\
\hline & 2 & 1 & 14.85 & 15.07 & 14.53 & 15.46 \\
\hline & & & & $J=$ & & \\
\hline \multirow{6}{*}{$\frac{1}{2}$} & 1 & 0 & 0.31 & 0.29 & 0.22 & 0.33 \\
\hline & 2 & 0 & 1.18 & 1.21 & 0.98 & 1.08 \\
\hline & 3 & 0 & 7.97 & 8.01 & 7.66 & 9.66 \\
\hline & 4 & 0 & 9.54 & 15.15 & 9.26 & 11.44 \\
\hline & 1 & 1 & 14.51 & 14.83 & 14.37 & 17.12 \\
\hline & 2 & 1 & 15.45 & 15.18 & 14.72 & 15.73 \\
\hline \multirow[t]{4}{*}{$\frac{3}{2}$} & 1 & 0 & 4.28 & 4.28 & 3.72 & 5.18 \\
\hline & 2 & 0 & 5.30 & 5.30 & 4.14 & 6.11 \\
\hline & 3 & 0 & 16.79 & 16.78 & 20.59 & 17.71 \\
\hline & 4 & 0 & 17.06 & 17.06 & 21.20 & 19.12 \\
\hline
\end{tabular}

${ }^{a}$ Close-coupled calculations.

${ }^{\mathrm{b}}$ Centrifugal-decoupled calculations.

${ }^{\mathrm{c}}$ Adiabatic-bender calculations.

${ }^{\mathrm{d} N o m i n a l}$ total angular momentum projection quantum number in body frame.

${ }^{\mathrm{e}}$ Adiabatic bender state index.

${ }^{\mathrm{f}}$ Nominal stretching quantum number; equal to the number of radial nodes in the vibrational wavefunction. (See Figs. 3-5 of Ref. 17.)

${ }^{g}$ Parity. Note that for a given parity, which is related to the index $\xi$ by $\pi$ $=\xi(-1)^{J-1 / 2}$, there occur two closely spaced levels.

$P \omega \mathrm{BF}$ basis can be expressed in terms of $\mu_{20} \cdot{ }^{18}$ The rotational line strength factors can be obtained in a similar fashion to the calculation of intensity factors in diatomic molecules. ${ }^{40}$

Two types of transitions need to be considered here, namely $P^{\prime}=\frac{1}{2} \leftarrow P^{\prime \prime}=\frac{1}{2}$ and $P^{\prime}=\frac{3}{2} \leftarrow P^{\prime \prime}=\frac{1}{2}$ bands. As in the case of diatomic transitions, the rotational line strength factors for the latter appear as a product of vibrational and rotational factors:

TABLE II. Vibrational band origins and rotational constants $\left(\mathrm{cm}^{-1}\right)$ in the effective Hamiltonian for the NO-Ne complex. ${ }^{a}$

\begin{tabular}{cccc}
\hline \hline Band & $E_{v_{b} v_{s} P}$ & $B$ & $D / 10^{-5}$ \\
\hline$A$ & 0 & 0.1070 & 1.75 \\
& 0 & 0.1130 & \\
$B$ & 4.051 & 0.1045 & 2.83 \\
& 3.83 & 0.1100 & 1.78 \\
$C$ & 8.034 & 0.0994 & 7.48 \\
& 8.54 & 0.0995 & \\
$D$ & 14.322 & 0.0850 & \\
& 15.18 & 0.0780 & \\
\hline \hline
\end{tabular}

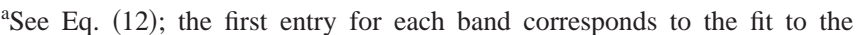
calculated level positions while the second entry is a best fit to the experimental spectra. The number of digits given indicates the uncertainty in the fit to the experimental spectra (see Ref. 17).
TABLE III. $\omega$-splitting constants $\left(\mathrm{cm}^{-1}\right)$ for the effective Hamiltonian for the NO-Ne complex. ${ }^{\mathrm{a}}$

\begin{tabular}{clcc}
\hline \hline Band & $V_{0}$ & $V_{2} / 10^{-3}$ & $V_{4} / 10^{-5}$ \\
\hline$A$ & 0.422 & 6.94 & -2.22 \\
& 0.33 & 6.80 & -5.74 \\
$B$ & 0.504 & 1.34 & \\
& 0.45 & 1.0 & -5.35 \\
$C$ & 0.743 & 6.0 & -1.17 \\
& 0.59 & -1.0 & \\
$D$ & 0.259 & 18.83 & \\
& 0.27 & 25.0 &
\end{tabular}

a See Eq. (12); the first entry for each band corresponds to the fit to the calculated level positions while the second entry is a best fit to the experimental spectra. The number of digits given indicates the uncertainty in the fit to the experimental spectra (see Ref. 17). We find (see last paragraph of Sec. IV) that the fit to the calculated energies leads to negligibly small values for the odd coefficients in Eq. (12) $\left(V_{1}\right.$ and $\left.V_{3}\right)$.

$$
\begin{aligned}
& S\left(J^{\prime} P^{\prime}=3 / 2, n^{\prime} v^{\prime} ; J^{\prime \prime} P^{\prime \prime}=1 / 2, n^{\prime \prime} v^{\prime \prime}\right) \\
& \quad=\left(2 J^{\prime}+1\right)\left(2 J^{\prime \prime}+1\right)|\mu|^{2}\left(\begin{array}{ccc}
J^{\prime} & 1 & J^{\prime \prime} \\
\frac{3}{2} & -1 & -\frac{1}{2}
\end{array}\right)^{2},
\end{aligned}
$$

where the transition dipole moment squared is given by an integral involving the $\mathrm{AB}$ vibrational wavefunctions for the initial and final states, namely, ${ }^{18}$

$$
|\mu|^{2}=\mu_{20}^{2}\left|\int \chi_{P^{\prime} n^{\prime}}^{v^{\prime}}(R) \mu_{n^{\prime} n^{\prime \prime}}^{(3)}(R) \chi_{P^{\prime \prime} n^{\prime \prime}}^{v^{\prime \prime}}(R) d R\right|^{2} .
$$

The explicit expression for $\mu_{n^{\prime} n^{\prime \prime}}^{(3)}$ has been given previously. ${ }^{18}$ We further remember that radiative transitions are allowed only between states of opposite parity $\left[\xi^{\prime}=\xi^{\prime \prime}\right.$ for $\Delta J= \pm 1$ and $\xi^{\prime}=-\xi^{\prime \prime}$ for $\left.\Delta J=0\right]$.

The rotational line strength factors for the $P^{\prime}=\frac{1}{2} \leftarrow P^{\prime \prime}$ $=\frac{1}{2}$ bands can be written as

$S\left(J^{\prime} P^{\prime}=1 / 2, n^{\prime} v^{\prime} ; J^{\prime \prime} P^{\prime \prime}=1 / 2, n^{\prime \prime} v^{\prime \prime}\right)$

$$
\begin{aligned}
= & \left(2 J^{\prime}+1\right)\left(2 J^{\prime \prime}+1\right) \mid\left(\begin{array}{ccc}
J^{\prime} & 1 & J^{\prime \prime} \\
\frac{1}{2} & 0 & -\frac{1}{2}
\end{array}\right) \mu^{\|} \\
& +\left.\xi^{\prime}\left(\begin{array}{ccc}
J^{\prime} & 1 & J^{\prime \prime} \\
-\frac{1}{2} & 1 & -\frac{1}{2}
\end{array}\right) \mu^{\perp}\right|^{2},
\end{aligned}
$$

TABLE IV. $P$-type doubling constants $\left(\mathrm{cm}^{-1}\right)$ for the effective Hamiltonian for the NO-Ne complex. ${ }^{\text {a }}$

\begin{tabular}{ccc}
\hline \hline Band & $C_{0} / 10^{-2}$ & $C_{1} / 10^{-3}$ \\
\hline$A$ & $0.60 / 0.81$ & $1.85 / 5.28$ \\
& 0.4 & 6.0 \\
$B$ & $0.15 / 0.39$ & $-0.14 /-0.33$ \\
& 0.0 & 0.0 \\
$C$ & $-1.43 /-2.44$ & $0.00 / 0.00$ \\
& 2.0 & 2.0 \\
$D$ & $-16.46 /-11.75$ & $1.32 / 1.11$ \\
& -13.0 & 0.0 \\
\hline
\end{tabular}

a See Eq. (12); for each band, the two entries on the first line correspond to the fit to the calculated level positions with, respectively $\omega=\frac{1}{2}$ and $\omega=-\frac{1}{2}$. The second entry is a best fit to the experimental spectra. The number of digits given indicates the uncertainty in the fit to the experimental spectra (see Ref. 17). 
where explicit expressions for the parallel (II) and perpendicular $(\perp)$ transition moments are given in our earlier paper. ${ }^{18}$ Thus, for the $P^{\prime}=\frac{1}{2} \leftarrow P^{\prime \prime}=\frac{1}{2}$ bands there will be interference between parallel and perpendicular transition moments. Consequently, the line strength factors cannot be separated into a product of rotational and vibrational factors.

The parallel transition moment leads to a weak $Q$-branch, while the perpendicular transition moment yields a strong $Q$-branch. Thus, the relative magnitudes and phase of the two terms in Eq. (16) will dictate the strength of the $Q$-branches in the $P^{\prime}=\frac{1}{2} \leftarrow P^{\prime \prime}=\frac{1}{2}$ transitions. However, we expect the perpendicular transition moments to be small for bands with $\omega^{\prime}=\omega^{18}$

Equations (14) and (16) were used, with the NO-Ne AB eigenfunctions expressed in the signed- $P \omega \mathrm{BF}$ basis [Eq. (4)] and the appropriate $\mathrm{AB}$ vibrational wavefunctions, to calculate the rotational line strength factors for transitions to the $n^{\prime}=1-4, P^{\prime}=\frac{1}{2}$ and $n^{\prime}=1-2, P^{\prime}=\frac{3}{2}$ bend-stretch levels of $\mathrm{NO}(v=2)-\mathrm{Ne}$. Since the PESs were calculated for a fixed value of the NO internuclear separation, the bend-stretch wavefunctions of the complex were taken to be independent of the vibrational quantum number of the NO moiety. In this case, the perpendicular transition moment is identically zero for $P^{\prime}=\frac{1}{2} \leftarrow P^{\prime \prime}=\frac{1}{2}$ bands with $n^{\prime}=n^{\prime \prime}$ (bands A and D).

\section{COMPARISON OF PREDICTED AND EXPERIMENTAL SPECTRA}

With the rotational line strength factors given in Sec. V and the positions of the energy levels of the complex from the $\mathrm{CC}$ calculations, we can predict the rotational band contours of the features seen in the experimental spectra, which is described in the accompanying article. To simulate these bands, we first assume that the $n=1$ and $2, P=\frac{1}{2}$ levels in the $\mathrm{NO}(X, v=0)-A r$ complex are populated thermally, at temperature $T \cong 1 \mathrm{~K}$. The spectrum is obtained by summing over all initially populated levels, and all allowed final levels, for transitions to the $n=1-4, P=\frac{1}{2}$ and $n=1-4, P=\frac{3}{2}$ levels of the $\mathrm{NO}(X, v=2)-\mathrm{Ar}$ complex. In addition, for a more direct comparison with experiment, we convolute the simulated stick spectrum with an assumed Gaussian linewidth, as described in our earlier publication. ${ }^{18}$

Figure 4 shows the predicted and experimental spectra corresponding to band A. Here, three different calculated spectra (labeled a, c, and d) are compared with the experimental trace (labeled b) from the preceding article. ${ }^{17}$ The spectrum based on the $a b$ initio calculations is displayed as trace c. Because the $a b$ initio PES calculations were at a fixed value of the NO bond distance, it is impossible to determine the shift of the $v=2 \leftarrow 0$ bands relative to the band origin of this transition in the isolated NO molecule. Consequently, the position of the $Q_{ \pm}^{ \pm}$branch was taken to lie at the experimental band origin $\left(3724.02 \mathrm{~cm}^{-1}\right.$, see Ref. 17). This also defines the origin for the positions of the ab initio predictions of bands $\mathrm{B}, \mathrm{C}$, and $\mathrm{D}$ (to be discussed later).

Spectrum $d$ was generated by fitting the bound-state energies of the $a b$ initio calculation to Eq. (12) with the parameters given in Table II-IV, under the assumption that that the intensity arises from a purely parallel transition [the $\mu^{\perp}$ transition moment from Eq. (16) was set equal to zero] and that

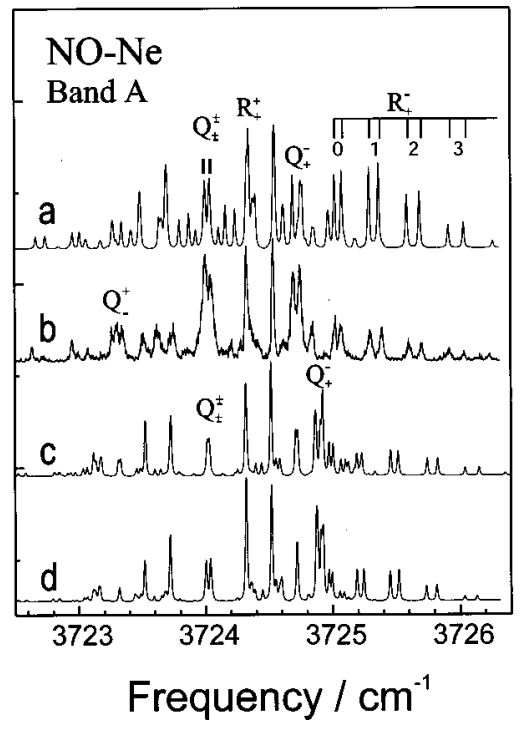

FIG. 4. Comparison of predicted and experimental spectra of the lowest $\mathrm{NO}(X, v=2 \leftarrow 0)$ overtone transition in the NO-Ne complex (band A), which corresponds to excitation of the $P=\frac{1}{2}, n=1$ and 2 van der Waals states in the vibrationally excited complex (see Fig. 3). The $v=2 \leftarrow 0$ band origin in the isolated NO molecule lies at $3723.85 \mathrm{~cm}^{-1}$ (Ref. 46). Trace a is the spectrum generated using Eq. (12) and the optimized sets of parameters from Tables II-IV. Trace $b$ is the experimental spectrum from Ref. 17. Trace $\mathrm{c}$ is the simulation from the $a b$ initio calculation. Trace $\mathrm{d}$ is the simulation using Eq. (12) with parameters corresponding to the best fit to the $a b$ initio energy levels (Tables II-IV). The assignment of the features in the experimental spectrum is taken from Ref. 17. The ordering of the traces is done so that the $a b$ initio and optimized theoretical simulations can both be compared most directly with experiment.

the Franck-Condon factor is constant. Here, too, we assumed that the position of the $Q_{ \pm}^{ \pm}$band lies at the experimental band origin

The overall agreement of traces $\mathrm{c}$ and $\mathrm{d}$ is excellent, although small differences in the intensities are observed. Comparison of either of these traces with experiment reveals good agreement in the overall rotational structure, but several important deviations in the line positions. Using the heuristic Hamiltonian of Eq. (12), we were able to improve the agreement with the observed spectrum by slight changes in the parameters. The resulting parameters, obtained by a leastsquares fit, are shown in Tables II-IV. The number of digits given indicates the uncertainty in the fit. ${ }^{17}$ The centrifugal distortion constant $D$ in Eq. (12) could be determined reliably only for the fit to the ab initio level positions (see Sec. $\mathrm{V})$, where precise values for each of the rotational levels are available.

The spectrum resulting from the best fit parameters in the Hamiltonian of Eq. (12) is shown as trace a in Fig. 4. In carrying out the fit we assumed that there is no difference in the parameters describing the $\mathrm{NO}(v=0)-\mathrm{Ne}$ and $\mathrm{NO}(v$ $=2)-\mathrm{Ne}$ levels. This approximation was made also in the determination of the spectra from the $a b$ initio level positions and intensities, as well as in our earlier investigation of the NO-Ar complex. ${ }^{18}$

Also marked in Fig. 4 are the position of several rotational branches. Due to the splitting of the rotational levels, we find four different types of branches corresponding to transitions with $\Delta \omega=0$ and \pm 1 . The superscript and subscript indicate, respectively, $\omega^{\prime}$ and $\omega^{\prime \prime}$. Since the parity 


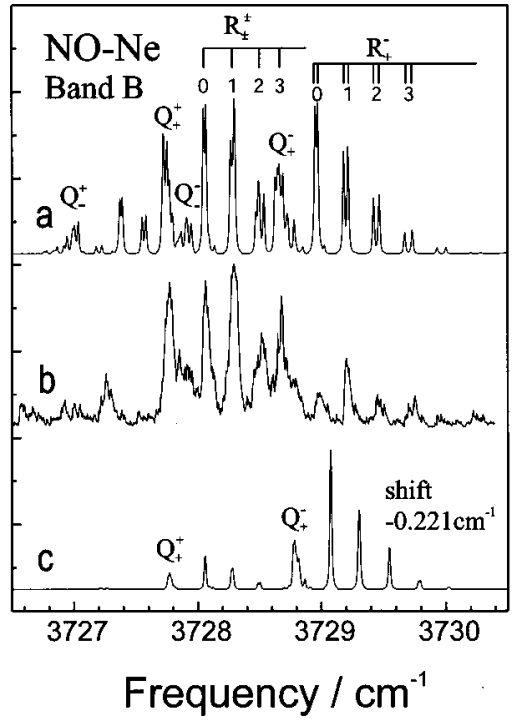

FIG. 5. Comparison of predicted and experimental spectra of the second $\mathrm{NO}(X, v=2 \leftarrow 0)$ overtone transition in the NO-Ne complex (band B), which corresponds to excitation of the $P=\frac{3}{2}, n=1$ and 2 van der Waals states in the vibrationally excited complex (see Fig. 3). The $v=2 \leftarrow 0$ band origin in the isolated NO molecule lies at $3723.85 \mathrm{~cm}^{-1}$ (Ref. 46). Trace a is the spectrum generated using Eq. (12) and the optimized sets of parameters from Tables II-IV. Trace b is the experimental spectrum from Ref. 17. Trace $\mathrm{c}$ is the simulation from the $a b$ initio calculation, shifted by 0.221 $\mathrm{cm}^{-1}$ to lower wave number. The assignment of the features in the experimental spectrum is taken from Ref. 17. The ordering of the traces is done so that the $a b$ initio and optimized theoretical simulations can both be compared most directly with experiment.

must change in the transition, the symmetry index $\xi$ changes for $Q$-branch lines but is conserved for the $P$ - and $R$-branch lines. The position of the various $Q$-branches reflects directly the $\omega$-splitting in the upper and lower states while the splittings of the lines in the $R_{+}^{-}$branch are an indication of the magnitude of the $P$-type doubling. Comparison with trace $c$ shows that the $a b$ initio calculation predicts too large a splitting between the different $Q$ branches.

Also, examination of the $R_{+}^{-}$branch shows that the predicted rotational constant is slightly too small (see Table II). This is understandable. Because the ab initio calculation does not completely recover the correlation energy, the dispersion energy is too small. Hence, the well in the potential is too shallow and its minimum occurs at too large a value of $R$. Finally, we see that the predicted splitting between the $R_{+}^{-}$ branch lines is slightly too small. This splitting is a reflection of the $P$-type doubling and its $J$ dependence. As $J$ increases, the splitting is dominated by the $C_{1}$ term, and is given, to a good approximation, by the sum of the $C_{1}$ terms for each parity. We see from the comparison of trace a and $\mathrm{c}$ that the level positions predicted by the ab initio calculations slightly underestimate this splitting, and hence the size of $C_{1}$ (Table IV).

Finally, the constant $P$-type doubling term $\left(C_{0}\right)$ in Eq. (12) is responsible for the splitting of the $Q_{+}^{ \pm}$branches. Because this splitting is poorly resolved in the experimental spectra, this constant will not be well determined by the fit to experiment.

Figures 5-7 display similar comparisons for bands B, C,

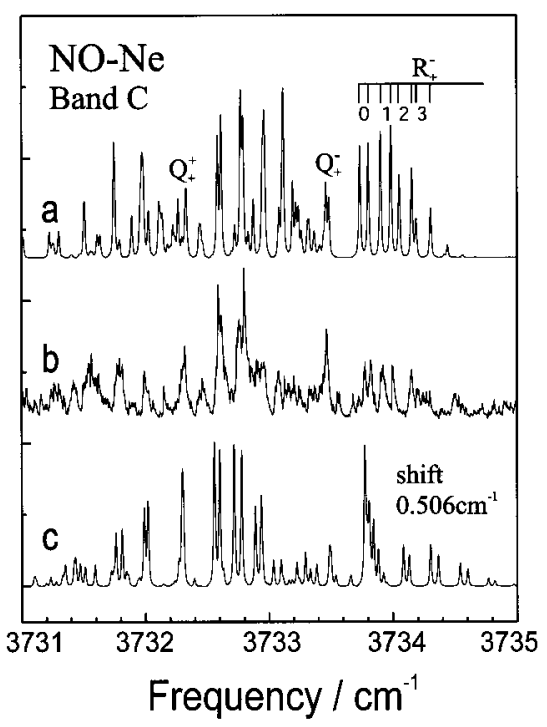

FIG. 6. Comparison of predicted and experimental spectra of the third $\mathrm{NO}(X, v=2 \leftarrow 0)$ overtone transition in the $\mathrm{NO}-\mathrm{Ne}$ complex (band $\mathrm{C}$ ), which corresponds to excitation of the $P=\frac{1}{2}, n=3$ and 4 van der Waals states in the vibrationally excited complex (see Fig. 3). The $v=2 \leftarrow 0$ band origin in the isolated NO molecule lies at $3723.85 \mathrm{~cm}^{-1}$ (Ref. 46). Trace a is the spectrum generated using Eq. (12) and the optimized sets of parameters from Tables II-IV. Trace $b$ is the experimental spectrum from Ref. 17. Trace $\mathrm{c}$ is the simulation from the $a b$ initio calculation, shifted by 0.506 $\mathrm{cm}^{-1}$ to higher wave number. The assignment of the features in the experimental spectrum is taken from Ref. 17. The ordering of the traces is done so that the $a b$ initio and optimized theoretical simulations can both be compared most directly with experiment.

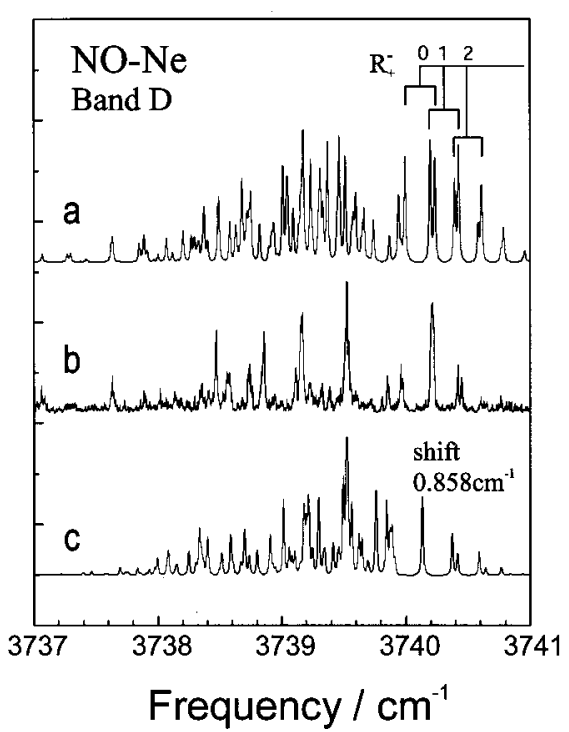

FIG. 7. Comparison of predicted and experimental spectra of the fourth $\mathrm{NO}(X, v=2 \leftarrow 0)$ overtone transition in the NO-Ne complex (band D), which corresponds to excitation of the first van der Waals stretching mode of the $P=\frac{1}{2}, n=1$ and 2 van der Waals states in the vibrationally excited complex (see Fig. 3). The $v=2 \leftarrow 0$ band origin in the isolated NO molecule lies at $3723.85 \mathrm{~cm}^{-1}$ (Ref. 46). Trace a is the spectrum generated using Eq. (12) and the optimized sets of parameters from Tables II-IV. Trace b is the experimental spectrum from Ref. 17. Trace $\mathrm{c}$ is the simulation from the $a b$ initio calculation, shifted by $0.858 \mathrm{~cm}^{-1}$ to higher wave number. The assignment of the features in the experimental spectrum is taken from Ref. 17. The ordering of the traces is done so that the ab initio and optimized theoretical simulations can both be compared most directly with experiment. 
and D. In all cases, the ab initio spectra are shifted slightly to best fit the position of the experimental spectra. This shift, which is always less than $1 \mathrm{~cm}^{-1}$, implies that the predicted origins of each band (the $E_{v_{b} v_{s}} P$ parameter in Table II), are slightly incorrect. Despite this fact, and although there are small deviations in the rotational line positions, we see that the agreement between the $a b$ initio calculations and experiment is, overall, excellent. For band B, as for band A, the predicted rotational constant is slightly too small. This is likely a consequence of the incomplete recovery of the correlation energy, as discussed earlier in this section.

Finally, we observe that for band D (Fig. 7), the simulation based on the heuristic Hamiltonian on Eq. (12) reproduces the experimental spectrum far less satisfactorily than we saw in the case of the other bands. Band D corresponds to excitation of one quantum of the van der Waals stretch. As we saw in our investigation of the similar NO-Ar complex, ${ }^{18}$ the bound-state wavefunction of this state is far more spread out than those for the lower states, which all have $v_{s}=0$. In this case, the heuristic Hamiltonian of Eq. (12), which assumes, implicitly, that the couplings can be modeled by values of the potential at a particular value of $R$, will fail, because the true wavefunction samples a large range of values of $R$.

\section{CONCLUSION}

In this article we have used ab initio PESs, along with a sophisticated treatment of the bound-state motion of the complex, to predict the vibrational excitation spectrum of the $\mathrm{NO}(X)-\mathrm{Ne}$ complex. The agreement with new experimental information, based on REMPI detection of vibrationally excited complexes, is outstanding, both in terms of the absolute spacing of the vibrational bands and the spacing and intensity of the rotational lines. Since the ro-vibronic wavefunctions of the initial and final states of the complex sample a rather extended region of the $\mathrm{NeNO}(X)$ PESs, this good agreement attests to the accuracy of the ab initio PESs. An improved fit to the experimental spectra was obtained, based on a heuristic Hamiltonian which includes the important couplings and splitting, with parameters derived from the $a b$ initio calculations.

Before the work of Meyer and co-workers, ${ }^{17,47}$ inelastic scattering studies provided the major source of experimental information on the interaction of $\mathrm{NO}$ with noble gases. The excellent agreement obtained here, and in our earlier investigation of the NO-Ar complex, ${ }^{18}$ suggests that current $a b$ initio methodology is sufficiently sophisticated to provide accurate PESs for these systems, at least in the region probed by the bound-state measurements of Meyer and co-workers. Consequently, deviations between the predictions of the same PESs and scattering studies may indicate residual systematic errors in these latter experiments.

\section{ACKNOWLEDGMENTS}

M.A., H.M., and P.D. are grateful to the U.S. National Science Foundation for its support under Grant Nos. CHE9971810 (to M.H.A. and P.J.D.) and CHE-9707670 (to H.M.). P.S. would like to thank the EPSRC (UK) for a Se- nior Research Assistantship at Durham University. T.W. wishes to thank the EPSRC (UK) for the award of an Advanced Fellowship and for the provision of computer time at the Rutherford Appleton Laboratories. E.P.F.L. is grateful for the support of the Research Grant Council (RGC) of the Hong Kong Special Administration Region (HKSAR) and the Research Committee of the Hong Kong Polytechnic University. Finally, the authors wish to thank Brian Howard for a copy of unpublished material from the thesis of Paul Mills (Ref. 48).

${ }^{1}$ For a comprehensive recent review, see P. J. Dagdigian, in Dynamics and Kinetics of Small Radicals, edited by K. Liu and A. F. Wagner (World Scientific, Singapore, 1995), Part I, p. 315.

${ }^{2}$ H. H. W. Thuis, S. Stolte, J. Reuss, J. J. H. van der Biesen, and C. C. H. van der Meijdenberg, Chem. Phys. 52, 211 (1980).

${ }^{3}$ J. M. Hutson, in Advances in Molecular Vibrations and Collision Dynamics, edited by J. M. Bowman and M. A. Ratner (JAI, Greenwich, CT, 1991), Vol. 1A, p. 1.

${ }^{4}$ M. T. Berry, R. A. Loomis, L. C. Giancarlo, and M. I. Lester, J. Chem. Phys. 96, 7890 (1992).

${ }^{5}$ M. I. Lester, S. E. Choi, L. C. Giancarlo, and R. W. Randall, Faraday Discuss. 97, 365 (1994).

${ }^{6}$ M. I. Lester, C.-C. Chuang, P. M. Andrews, M. Yang, and M. H. Alexander, Faraday Discuss. 102, 311 (1995).

${ }^{7}$ For a good review, see M. C. Heaven, J. Phys. Chem. 97, 8567 (1993).

${ }^{8}$ B.-C. Chang, L. Yu, D. Cullin, B. Rehfuss, J. Williamson, T. A. Miller, W. M. Fawzy, X. Zheng, S. Fei, and M. Heaven, J. Chem. Phys. 95, 7086 (1991).

${ }^{9}$ B.-C. Chang, J. R. Dunlop, and T. A. Miller, Chem. Phys. Lett. 207, 55 (1993).

${ }^{10}$ B.-C. Chang, J. R. Dunlop, J. W. Williamson, T. A. Miller, and M. C. Heaven, Chem. Phys. Lett. 207, 62 (1993).

${ }^{11}$ C. C. Carter, H.-S. Lee, A. B. McCoy, and T. A. Miller, J. Mol. Struct. 1, 525 (2000).

${ }^{12}$ B. J. Howard, C. M. Western, and P. D. Mills, Faraday Discuss. Chem. Soc. 73, 129 (1982).

${ }^{13}$ P. D. A. Mills, C. M. Western, and B. J. Howard, J. Phys. Chem. 90, 4961 (1986).

${ }^{14}$ Y. Kim, K. Patton, J. Fleniken, and H. Meyer, Chem. Phys. Lett. 318, 522 (2000).

${ }^{15}$ J. C. Miller and W.-C. Cheng, J. Phys. Chem. 96, 2573 (1985).

${ }^{16}$ J. Fleniken, Y. Kim, and H. Meyer, J. Chem. Phys. 109, 8940 (1998),

${ }^{17}$ Y. Kim, J. Fleniken, and H. Meyer, J. Chem. Phys. 114, 5577 (2001), preceding paper.

${ }^{18}$ Y. Kim, J. Fleniken, H. Meyer, M. H. Alexander, and P. J. Dagdigian, J. Chem. Phys. 113, 73 (2000).

${ }^{19}$ M. H. Alexander, Chem. Phys. 92, 337 (1985).

${ }^{20}$ P. J. Knowles, C. Hampel, and H.-J. Werner, J. Chem. Phys. 99, 5219 (1993); ibid. 112, 3106 (2000).

${ }^{21}$ MOLPRO is a package of $a b$ initio programs written by H.-J. Werner and P. J. Knowles, with contributions from J. Almlöf, R. D. Amos, M. J. O. Deegan, S. T. Elbert, C. Hampel, W. Meyer, K. Peterson, E. A. Reinsch, R. Pitzer, A. Stone, and P. R. Taylor.

${ }^{22}$ GAUSSIAN 98 is the latest in the Gaussian series of electronic structure programs. For more information, see the web site http:// www.gaussian.com/g98broc.htm

${ }^{23}$ S. F. Boys and F. Bernardi, Mol. Phys. 19, 553 (1970).

${ }^{24}$ W. Gautschi, ACM Trans. Math. Softw. 20, 21 (1994); 24, 355 (1998).

${ }^{25}$ T.-S. Ho and H. Rabitz, J. Chem. Phys. 104, 2584 (1996).

${ }^{26}$ T. Hollebeek, T.-S. Ho, and H. Rabitz, Annu. Rev. Phys. Chem. 50, 537 1999).

${ }^{27}$ P. Soldán and J. M. Hutson, J. Chem. Phys. 112, 4415 (2000).

${ }^{28}$ D. M. Brink and G. R. Satchler, Angular Momentum, 2nd ed. (Clarendon, Oxford, 1968).

${ }^{29}$ M. H. Alexander, J. Chem. Phys. 111, 7426 (1999); M. H. Alexander and S. Stolte, ibid. 112, 8017 (2000).

${ }^{30}$ E. P. F. Lee and T. G. Wright, J. Chem. Phys. 109, 157 (1998).

${ }^{31}$ J. Klos, G. Chalasinski, M. T. Berry, R. Bukowski, and S. M. Cybulski, J. Chem. Phys. 112, 2195 (2000).

${ }^{32}$ M. H. Alexander, J. Chem. Phys. 111, 7435 (1999). 
${ }^{33}$ G. C. Corey and M. H. Alexander, J. Chem. Phys. 85, 5652 (1986).

${ }^{34}$ M.-L. Dubernet, P. A. Tuckey, and J. M. Hutson, Chem. Phys. Lett. 193, 355 (1992).

${ }^{35}$ M.-L. Dubernet, D. Flower, and J. M. Hutson, J. Chem. Phys. 94, 7602 (1991).

${ }^{36}$ S. L. Holmgren, M. Waldman, and W. Klemperer, J. Chem. Phys. 67, 4414 (1977).

${ }^{37}$ M. H. Alexander, S. Gregurick, and P. J. Dagdigian, J. Chem. Phys. 101, 2887 (1994).

${ }^{38}$ M. H. Alexander, S. Gregurick, P. J. Dagdigian, G. W. Lemire, M. J. McQuaid, and R. C. Sausa, J. Chem. Phys. 101, 4547 (1994).

${ }^{39}$ W. H. Green, Jr. and M. I. Lester, J. Chem. Phys. 96, 2573 (1992).

${ }^{40}$ R. N. Zare, Angular Momentum (Wiley, New York, 1988).

${ }^{41}$ H. Lefebvre-Brion and R. W. Field, Perturbations in the Spectra of Diatomic Molecules (Academic, New York, 1986).
${ }^{42}$ M. Yang, M. H. Alexander, S. Gregurick, and P. J. Dagdigian, J. Chem. Phys. 102, 2413 (1995).

${ }^{43}$ M. Yang and M. H. Alexander, J. Chem. Phys. 103, 3400 (1995).

${ }^{44}$ I. P. Hamilton and J. C. Light, J. Chem. Phys. 84, 306 (1986).

${ }^{45}$ HIBRIDON is a package of programs for the time-independent quantum treatment of inelastic collisions and photodissociation written by $\mathrm{M}$. $\mathrm{H}$. Alexander, D. E. Manolopoulos, H.-J. Werner, and B. Follmeg, with contributions by P. F. Vohralik, D. Lemoine, G. Corey, B. Johnson, T. Orlikowski, W. Kearney, A. Berning, A. Degli-Esposti, C. Rist, and P. Dagdigian. More information and/or a copy of the code can be obtained from the website http://www-mha.umd.edu/ mha/hibridon

${ }^{46}$ C. Amiot, R. Bacis, and G. Guelachvili, Can. J. Phys. 56, 251 (1978).

${ }^{47}$ Y. Kim, K. Patton, J. Fleniken, and H. Meyer, Chem. Phys. Lett. 318, 522 (2000).

${ }^{48}$ P. D. A. Mills, D. Phil. thesis, University of Oxford (1986). 